\title{
Screening for Subsequent Pancreatic Cancer in Childhood Cancer Survivors is Needed. A Case Report
}

Lorna Zadravec Zaletel", Miha Cavlek and Mojca Gjidera

Department of Radiation Oncology, Institute of Oncology, Zaloška 2, 1000, Ljubljana, Slovenia

*Corresponding author: Lorna Zadravec Zaletel, Department of Radiation Oncology, Institute of Oncology, Zaloška 2, 1000, Ljubljana, Slovenia, Tel: 0038641436575; E-mail: Izaletel@onko-i.si

Received date: November 14, 2017; Accepted date: November 20, 2017; Published date: November 27, 2017

Copyright: (c) 2017 Zadravec Zaletel L, et al. This is an open-access article distributed under the terms of the Creative Commons Attribution License, which permits unrestricted use, distribution, and reproduction in any medium, provided the original author and source are credited.

\begin{abstract}
Introduction: The survival of children with cancer has improved considerably over the last decades which have led to a growing number of long term survivors. One of the most serious adverse outcomes in this population is subsequent primary neoplasms with the risk as high as six-fold over expected. The relative risk for subsequent pancreatic cancer in childhood cancer survivors is high, especially after subdiafragmatic radiotherapy and/or chemotherapy with alkylating agents. The survival of patient with advanced pancreatic cancer is very low, and early diagnosis is of vital importance.
\end{abstract}

Case report: We present a female patient, treated for Ewing sarcoma of thoracic wall with multimodal treatment at the age of 5 . She received chemotherapy containing a high dose of Cyclophosphamide and postoperative radiotherapy to the left hemithorax with the dose of $15 \mathrm{~Gy}$ followed by local boost to the tumour bed. She had regular follow-ups at the Late-effect clinic at the Institute of Oncology in Ljubljana. Well differentiated papillary cancer of thyroid with nodal metastases was diagnosed during a regular neck ultrasound 26 years after the completion of cancer treatment. Two years later, at the age of 33 , she presented with abdominal distension, inappetence and diffuse liver metastases with ascites were found by abdominal ultrasound. Tumour marker CA 19-9 was high, but pancreas was morphologically normal on the ultrasound and on abdominal helical CT. Cytological test of ascites and hepatic metastases showed poorly differentiated adenocarcinoma. The disease progressed very fast and she died a month after the appearance of the first symptom. The autopsy revealed poorly differentiated adenocarcinoma of the pancreatic head and body with multiple metastases in abdominal organs and lungs.

Conclusion: It would be reasonable to introduce the same guidelines for screening of subsequent pancreatic cancer in childhood cancer survivors at high risk for subsequent pancreas cancer as proposed for patients with increased risk for familial pancreatic cancer.

Keywords: Subsequent pancreas cancer; Childhood cancer survivor; Late effects; Subsequent primary neoplasm; Screening

Abbreviations SPN: Subsequent Primary Neoplasm; CCS: Childhood Cancer Survivor; RT: Radiotherapy; ChT: Chemotherapy; AA: Alkylating Agents; SPC: Subsequent Pancreatic Cancer

\section{Introduction}

The survival of children with cancer has improved considerably over the last decades which have led to a growing number of long term survivors. It is estimated that there are between 300,000 and 500,000 survivors of childhood cancer in Europe [1]. A significant proportion of childhood cancer survivors (CCS) suffer adverse health outcomes including premature mortality, compared with general population [2]. One of the most serious adverse outcomes is subsequent primary neoplasms (SPNs), with the risk as high as six-fold over expected [3-6].

The risk of developing a SPN of gastrointestinal tract is high. Tukenova et al observed 9.7-fold higher risk of SPNs in CCS in relation to general population in France and the United Kingdom. The relative risk of subsequent pancreatic cancer (SPC) in this cohort was 9.9 [7]. High risk of pancreatic cancer (odds ratio of 19) was observed in patients treated for Hodgkin's lymphoma with subdiafragmatic radiotherapy (RT) and > 6 cycles of chemotherapy (ChT) with alkylating agents (AA) [8]). A lower relative risk (3.6) of SPC was reported in adult patients with testicular cancer [9]. The survival of patients with advanced pancreatic cancer is very low, and early diagnosis is of vital importance [10]. To our knowledge no guidelines exist for the screening of pancreatic cancer in CCS who are at high risk for SPC.

Hereinafter, we describe the history of a female patient, treated for Ewing sarcoma of thoracic wall with multimodal treatment at the age of 5, who died of SPC with fulminant course 28 years after the treatment.

\section{Case Report}

Our female patient was treated in 1987, at the age of five for Ewing sarcoma of the posterior part of the left fifth rib with infiltration of adjacent soft tissue. Wide resection of the tumour was performed after 3 cycles of preoperative ChT according to T6 protocol. A total necrosis was found in microscopic examination. The patient received postoperative RT with the dose of $15 \mathrm{~Gy}$ in $1.5 \mathrm{~Gy}$ fractions to the left hemithorax with cobalt gamma rays through antero-posterior and postero-anterior field, followed by boost with electrons $(10 \mathrm{MeV})$ to the tumour bed at the upper half of left posterior thoracic wall with 14 
Citation: Zadravec Zaletel L, Cavlek M, Gjidera M (2017) Screening for Subsequent Pancreatic Cancer in Childhood Cancer Survivors is Needed. A Case Report. J Nucl Med Radiat Ther 8: 348. doi:10.4172/2155-9619.1000348

Page 2 of 4

Gy in 7 fractions. Postoperative ChT according to T2 protocol was administered for one and a half year. Cumulative doses of cytostatics were: $17500 \mathrm{mg} / \mathrm{m}^{2}$ of Cyclophosphamide, $370 \mathrm{mg} / \mathrm{m}^{2}$ of Adriablastine, $11000 \mathrm{mg} / \mathrm{m}^{2}$ of Dactinomycin, $48 \mathrm{mg} / \mathrm{m}^{2}$ of Vincristine, $75 \mathrm{mg} / \mathrm{m}^{2}$ of Bleomycine and $85 \mathrm{mg} / \mathrm{m}^{2}$ of MTX.

Following that, she had regular follow-ups, at first at the Paediatric clinic, and from the age of 16 at the Late-effect clinic at the Institute of Oncology in Ljubljana [11]. There, regular screening for secondary breast cancer and regular tests of cardiac and thyroid function were performed. In 2013, 2 years after the end of cancer treatment, at the age of 31, at a routine ultrasound of the neck, nodule $6 \mathrm{~mm}$ in diameter was found in the left thyroid lobe. A cytology test pointed to a papillary cancer. A total thyroidectomy and modified dissection of neck lymph nodes was done. Histology revealed well differentiated papillary cancer in the left thyroid lobe with lymph node metastases in the first three levels of left neck. After the surgery the patient underwent ablation of the thyroid tissue with radioactive iodine 131-I $(96 \mathrm{mCi})$.

A year later, fibroadenoma at the border of outer quadrants of the right breast was diagnosed by biopsy of the lesion seen on diagnostic MRI. In May 2015, at a regular control at the clinic for long-term follow-up she felt well, with no complaints, her clinical test was ok, blood test was scheduled in 3 months. However, one and a half months later she came back with medical reports from a regional hospital where laparoscopic gallbladder stone surgery had been planned but was not performed due to abdominal distension. Abdominal ultrasound found finely granulated liver and ascites. Abdominal CT revealed numerous liver metastases, moderate hydronephrosis of the left kidney, other abdominal organs, including pancreas, were morphologically normal (Figures 1 and 2). She complained because of abdominal distension, inappetence.

A physical examination showed that she was malnourished, WHO performance status was 2 , abdominal distension was seen, caused by extensive ascites. Fine needle aspiration of hepatic lesion was performed and cytological test showed poorly differentiated adenocarcinoma. In the cytology test of ascites, peritoneal carcinosis was found. Blood tests showed highly elevated levels of hepatic enzymes, direct bilirubin and abnormal tumour markers CEA (155, normal<4.7) and CA 19-9 $(2118$, normal<30). She was hospitalised at the Institute of Oncology and abdominal helical CT was performed, showing huge ascites, many hepatic metastases, largest $6.5 \mathrm{~cm}$ in diameter, marginally enlarged left paraaortic lymph nodes, enlargement of both ovaries. Pancreas looked normal, even pancreatic duct did not appear dilated on CT. Multidisciplinary team proposed symptomatic treatment because of poor performance status. Namely, her physical condition was worsening very quickly from day to day and she died 10 days after admission to the hospital. Autopsy was performed and revealed poorly differentiated adenocarcinoma of the pancreatic head and body, invading into peripancreatic soft tissue and the wall of stomach. Multiple metastases with massive lymphovascular invasion of lung, spleen, gallbladder, both suprarenal glands, mesentery, small bowel wall, uterus, adnexes and abdominal lymph nodes were found.

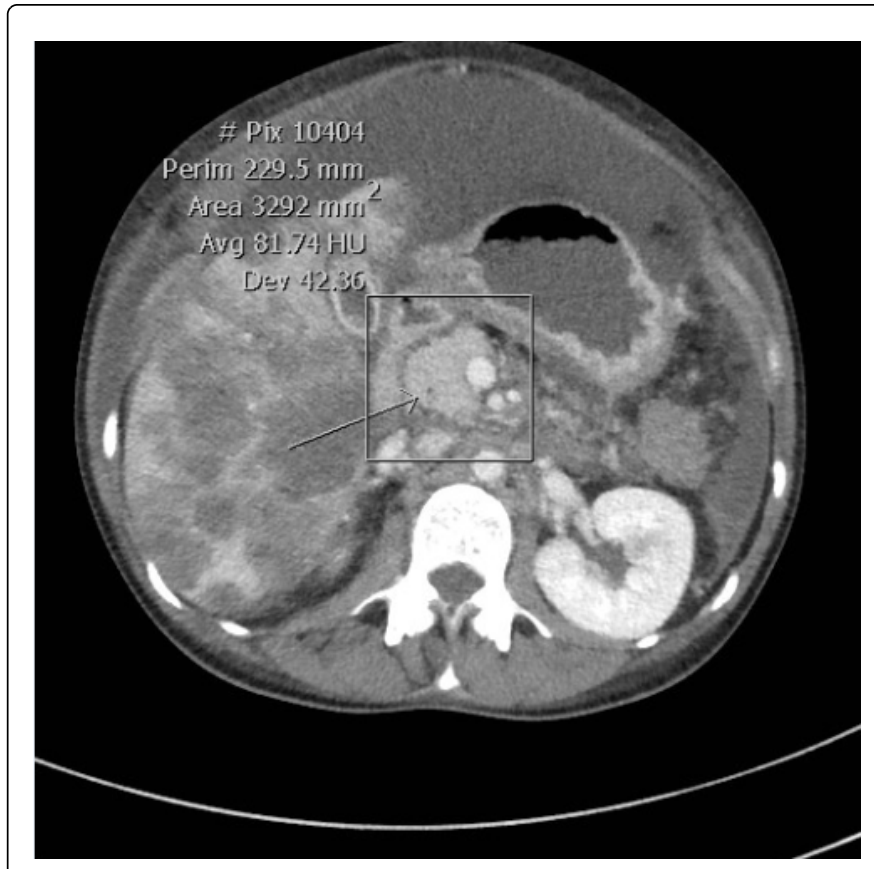

Figure 1: CT scan of the abdomen (transverse section). Pancreas of normal structure and size is seen. Pancreatic duct is not dilated (an arrow). Ascites and diffuse liver metastases are present.

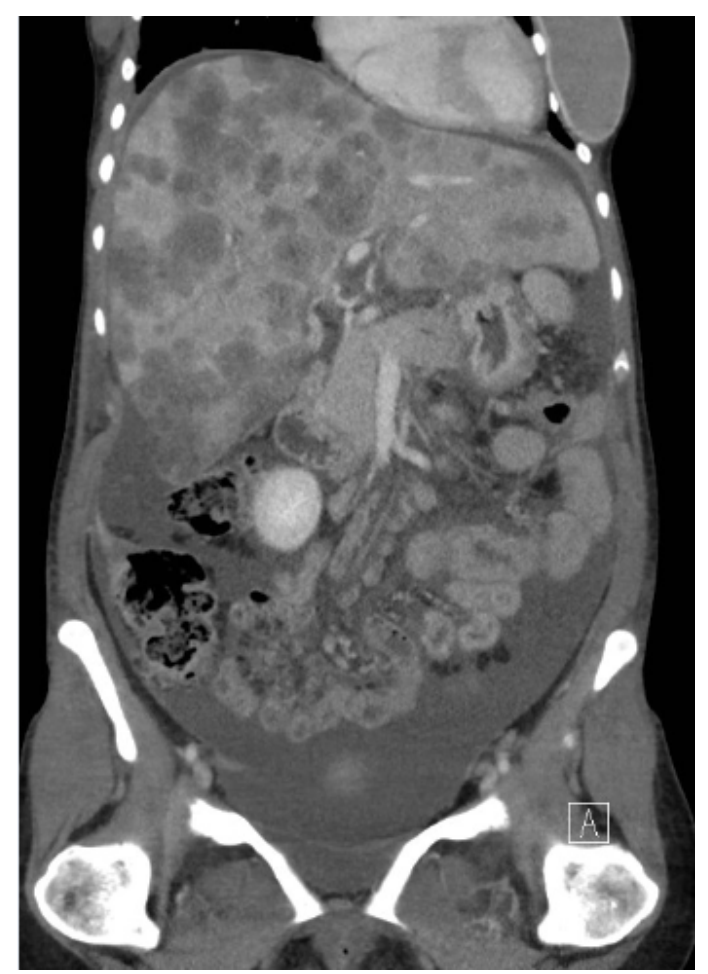

Figure 2: CT scan of the abdomen (coronal plane). Pancreas is of normal structure and size. Ascites and diffuse liver metastases are seen. 


\section{Discussion}

Our patient experienced a fulminant course of SPC and died a month after the appearance of the first, nonspecific, symptom of metastatic pancreas cancer. In childhood she underwent RT to the left hemithorax with the dose of 15 Gy and most likely at least upper part of the pancreas was in RT field. Beside that she received a high cumulative dose of AA Cyclophosphamide. According to the literature, RT and ChT with AA are important cause of SPC. In the report of Dores et al, SPC risk after treatment of Hodgkin lymphoma increased with increasing radiation dose to the pancreatic tumour location and increasing number of AA-containing cycles of ChT, especially high risk was observed in patients receiving $>3900 \mathrm{mg} / \mathrm{m}^{2}$ of cyclophosphamide (8). The odds ratio for patients treated with both subdiaphragmatic radiation ( $\geq 10 \mathrm{~Gy}$ ) and $\geq 6 \mathrm{AA}$-containing ChT cycles compared with patients with neither treatment was as high as 17.9. Hauptmann et al in the case-control study in testicular cancer patients reported that odds ratio of SPC increased linearly by 0.12 per Gy to the pancreas [12]. In the same study the risk increased with the number of ChT cycles of ChT with AA or platinum agents as well. Besides that, it is reported that CCS treated for Ewing sarcoma, Hodgkin lymphoma and bilateral retinoblastoma are at the highest risk for SPN $[5,13]$. Our patient got SPC after a relatively long latency period of 28 years after treatment for Ewing sarcoma. In other studies, median interval between the primary neoplasm and SPC was shorter, between 19 and 20.5 years [7,8].

Pancreatic cancer remains one of the deadliest cancers worldwide with a poor, five-year survival rate of $5 \%$ [10]. To improve the patient survival rate, early detection of pancreatic cancer is of vital importance. Multi-detector row computed tomography (MDCT) is very effective for detecting and staging of pancreas adenocarcinoma, its sensitivity being up to $90 \%$ for detection and an accuracy of $80 \%-90 \%$ for staging. It is favourably complemented by endoscopic ultrasound, which is more sensitive to the early detection of lesions, and allows relatively easy access to the pancreas for tissue diagnosis using fine needle aspiration [14]. Abdominal ultrasound is the most accessible, widely used modality and often used first to identify the cause of abdominal pain or other symptoms and signs. But the accuracy of conventional ultrasound for diagnosing pancreatic tumours is only $50-70 \%$ [15]. Widely used biomarker CA $19-9$ is not suitable for early diagnosis of pancreatic cancer because of its inadequate sensitivity and specificity, but maybe in the future effective biomarkers, like microRNA, in combination with imaging would provide an ideal approach for early detection of pancreatic cancer [16]. In our patient, SPC was neither detected by abdominal ultrasound nor on helical CT, performed a few days before death. Even pancreatic duct did not appear dilated on CT. At autopsy an area of $10 \mathrm{~cm}$ in diameter in pancreatic head and body was finely granulated and a nodular area of $2.5 \mathrm{~cm}$ inside that was found. There are very precise guidelines for the screening of some SPN in CCS, for example breast cancer and colon cancer (COG) [17], but to our knowledge, no guidelines exist for the screening of pancreatic cancer in this vulnerable population of patients. It would be reasonable to introduce the same guidelines for screening as proposed for patients with increased risk for familial pancreatic cancer. Namely, International Cancer of the Pancreas Screening Consortium agreed that initial screening for this population should include endoscopic ultrasonography and/or magnetic resonance cholangiopancreatography. Nevertheless, there was no consensus on the need for fine-needle aspiration to evaluate cysts, besides that, more evidence is needed, particularly for how to manage patients with detected lesions [18]. According to studies on SPC, the proposed age of CCS at which we should start the screening would be as early as 17 years after the end of treatment $[7,8]$.

Our patient suffered from subsequent well differentiated papillary cancer as well, most likely caused by RT. Small tumour was detected on routine ultrasound, but nodal metastases were already present. Thyroid cancers are beside breast cancers the most common SPN in CCS and are mostly well differentiated cancer with excellent prognosis [19]. Currently there is no consensus on the optimal surveillance strategy for thyroid cancer after radiotherapy in CCS. The use of ultrasound for early diagnosis of radiation-induced thyroid cancer may be suitable, nevertheless, routine ultrasound screening in irradiated CCS can actually not be recommended [20].

\section{Acknowledgement}

We thank for financial support to Slovenian Research Agency.

\section{References}

1. Mariotto AB, Rowland JH, Yabroff KR, Scoppa S, Hachey M, et al. (2009) Long-term survivors of childhood cancers in the United States. Cancer Epidemiol Biomark Prev 18: 1033-1040.

2. Armstrong GT, Liu Q, Yasui Y, Neglia JP, Leisenring W, et al. (2009) Late Mortality Among 5-Year Survivors of Childhood Cancer: A Summary From the Childhood Cancer Survivor Study. J Clin Oncol 27: 2328-2338.

3. Bhatia S, Sklar C (2002) Second cancers in survivors of childhood cancer. Nat Rev Cancer 2: 124-132.

4. Meadows AT (1988) Risk factors for second malignant neoplasms: report from the Late Effects Study Group. Bull Cancer 75: 125-130.

5. Friedman DL, Whitton J, Leisenring W, Mertens AC, Hammon S, et al. (2010) Subsequent Neoplasms in 5-Year Survivors of Childhood Cancer: The Childhood Cancer Survivor Study. J Natl Cancer Inst 102: 1083-1095.

6. Ng AK, Kenney LB, Gilbert ES, Travis LB (2010) Secondary malignancies across the age spectrum. Semin radiat oncol 20: 10 .

7. Tukenova M, Diallo I, Anderson H, Hawkins M, Garwicz S, et al. (2012) Second malignant neoplasms in digestive organs after childhood cancer: A cohort-nested case-control study. Int J Radiat Oncol Biol Phys 1: 383-90.

8. Dores GM, Curtis RE, van Leeuwen FE, Stovall M, Hall P, et al. (2014) Pancreatic cancer risk after treatment of Hodgkin Lymphoma. Annals Oncol 25: 2073-2079.

9. Travis LB, Fossa SD, Schonfeld SJ, McMaster ML, Lynch CF, et al. (2005) Second cancers among 40,576 testicular cancer patients: Focus on longterm survivors. J Natl Cancer Inst. 97:1354-1365.

10. Lee ES, Lee JM (2014) Imaging diagnosis of pancreatic cancer: A state-ofthe-art review. World J Gastroenterol『WJG 20: 7864-7877.

11. Jereb B (2000) Model for long-term follow-up of survivors of childhood cancer. Med Pediatr Oncol 34: 256-258.

12. Hauptmann M, Johannesen TB, Gilbert ES, Stovall M, van Leeuwen FE, et al. (2016) Increased pancreatic cancer risk following radiotherapy for testicular cancer. Br J Cancer 115: 901-908.

13. Vathaire de F, Hawkins M, Campbell S, Oberlin O, Raquin MA, et al. (1999) Second malignant neoplasms after a first cancer in childhood: Temporal pattern of risk according to type of treatment. Br Journal of Cancer 79: 1884-1893.

14. Kinney $\mathrm{T}$ (2010) Evidence-based imaging of pancreatic malignancies. Surg Clin North Am 90: 235-249.

15. Miura F, Takada T, Amano H, Yoshida M, Furui S, et al. (2006) Diagnosis of pancreatic cancer. HPB 8: 337-342.

16. Huang J, Liu J, Chen-Xiao K, Zhang X, Lee WNP, et al. (2016) Advance in microRNA as a potential biomarker for early detection of pancreatic cancer. Biomarker Research 4: 20. 
Citation: Zadravec Zaletel L, Cavlek M, Gjidera M (2017) Screening for Subsequent Pancreatic Cancer in Childhood Cancer Survivors is Needed. A Case Report. J Nucl Med Radiat Ther 8: 348. doi:10.4172/2155-9619.1000348

Page 4 of 4

17. Mulder RL., Kremer LCM, Hudson MM, Bhatia S, Landier W, et al (2013) Recommendations for Breast Cancer Surveillance for Female Childhood, Adolescent and Young Adult Cancer Survivors Treated with Chest Radiation: A Report from the International Late Effects of Childhood Cancer Guideline Harmonization Group. The Lancet Oncology 14: 621-629.

18. Canto MI, Harinck F, Hruban RH, Offerhaus GJ, Poley, et al. (2013) International Cancer of the Pancreas Screening (CAPS) Consortium summit on the management of patients with increased risk for familial pancreatic cancer Gut 62: 339-347.

19. Turcotte LM, Liu Q, Yasui Y, Arnold MA, Hammond S, et al. (2017) Temporal trends in treatment and subsequent neoplasm risk among fiveyear survivors of childhood cancer, 1970-2015. JAMA 317: 814-824.

20. Brignardello E, Felicetti F, Castiglione A, Gallo M, Maletta F, et al. (2016) Ultrasound surveillance for radiation-induced thyroid carcinoma in adult survivors of childhood cancer. Eur J Cancer 55: 74-80. 